\title{
Corrigendum
}

\section{Betaine alleviates hepatic lipid accumulation via enhancing hepatic lipid export and fatty acid oxidation in rats fed with a high-fat diet - CORRIGENDUM}

\author{
Li Xu, Danping Huang, Qiaoling Hu, Jing Wu, Yizhen Wang and Jie Feng* \\ (First published online: 29 April 2015) \\ doi:http://dx.doi.org/10.1017/S0007114515001130, Published by Cambridge University Press, April 2015
}

\section{Abstract}

In the abstract, these sentences (page 1, line 5) should be: 'Additionally, hepatic betaine-homocysteine methyltransferase concentration as well as its mRNA abundance and lecithin level were found increased $(P<0 \cdot 05)$ by betaine supplementation in both basal diet-fed rats and high-fat diet-fed rats. Betaine administration in high-fat diet-fed rats exhibited a higher $(P<0 \cdot 05)$ concentration of hepatic carnitine palmitoyltransferase 1 (CPT1) comparedwith high-fat diet-fed rats.'

\section{Materials and methods}

\section{Hepatic histology and hepatic lipid metabolites analysis}

In this paragraph, the last sentence (page 2, line 15) should be: 'Hepatic carnitine, carnitine palmitoyltransferase 1 (CPT1) and betaine-homocysteine methyltransferase (BHMT) concentration were determined by using ELISA kits (A\&D Company Limited, China) according to manufacturer's instructions.

The catalogue numbers of the ELISA kits: Rat CPT1 ELISA kit, code-CSB-E16442r; Rat BHMT ELISA kit, code-CSB-EL002693RA.

\section{Results}

Betaine elevated the concentration and the mRNA expression of betaine-homocysteine methyltransferase in the liver

In this paragraph, the first sentence (page 4, line 1) should be: 'Compared with groups without betaine administration (T1 and T3), betaine supplementation groups (T2 and T4) exhibited a significant increase $(P<0.05)$ in the concentration of BHMT in the liver (Fig. 3(a)). High-fat diet also elevated $(P<0.05)$ the concentration of BHMT when compared with rats fed with basal diet.'

Betaine increased PPARa gene expression and reversed the inhibition of carnitine palmitoyltransferase 1 gene expression induced by high-fat diet in the liver

In this paragraph, the second sentence (page 4, line 3) should be: "Betaine administration in rats fed with a high-fat diet exhibited a higher concentration of CPT1 $(P<0.05)$ compared with that of high-fat diet-fed rats (Fig. 4(a)).'

Betaine increased the concentration, gene and protein expression of fibroblast growth factor 21, and elevated the gene expression of AMP-activated protein kinase in the liver

In this paragraph, the first sentence (page 5 , line 1 ) should be: "The changes of betaine administration on the concentration, gene and protein expression of FGF21 in the liver are shown in Fig. 5(a)-(c).'

\section{Discussion}

In this part, the second paragraph the third sentence (page 6, line 18) should be: 'Significant increase in BHMT concentration was observed in betaine administration rats given either basal diet or high-fat diet, which was accompanied with the notable increase of BHMT mRNA abundance in the high-fat group.'

In this part, the second paragraph the sentence (page 6, line 6) should be: 'Our results showed that betaine administration elevated the levels of hepatic lecithin and serum lecithin and VLDL in high-fat diet-fed rats, which suggested that betaine reversed hepatic lipid accumulation by elevated lecithin and VLDL levels via increasing the expression and concentration of BHMT, thus enhancing hepatic lipid export. Besides, high-fat diet also increased the concentration of BHMT in the liver.'

In this part, the third paragraph the third sentence (page 6, line 6) should be: 'The results showed that betaine 
(a)

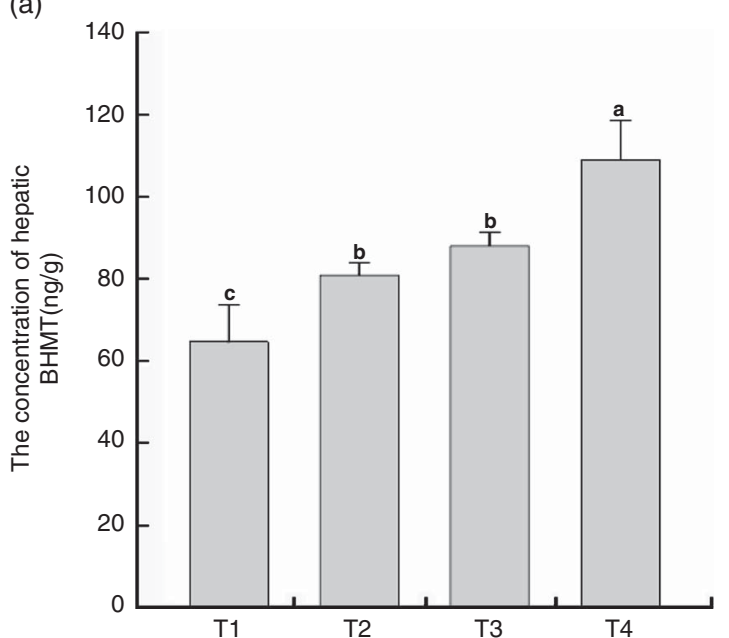

(b)

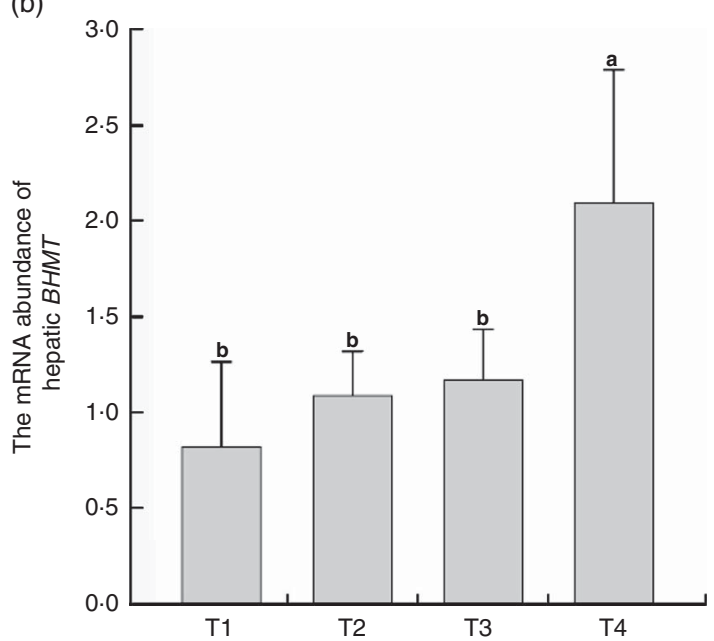

Fig. 3. (a) Effect of betaine on the concentration of betaine-homocysteine methyltransferase (BHMT) in the liver. (b) Effect of betaine on the mRNA abundance of BHMT in the liver. Values are means $(n 7)$, with standard deviations represented by vertical bars. ${ }^{a, b, c}$ Mean values with unlike letters were significantly different $(P<0.05)$. Groups: T1, basal diet; T2, basal diet with betaine administration; T3, high-fat diet; T4, high-fat diet with betaine administration.

(a)

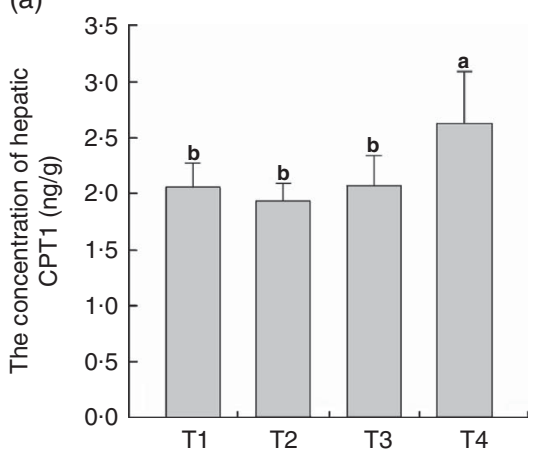

(b)

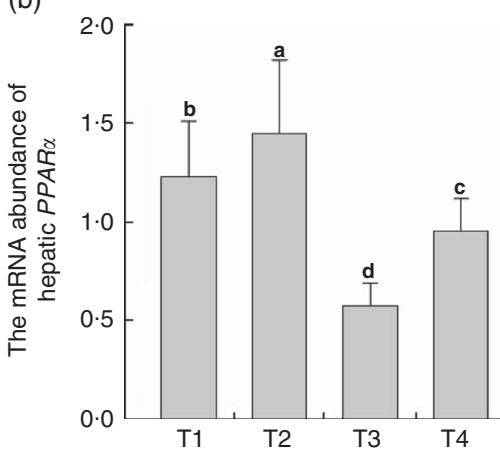

(c)

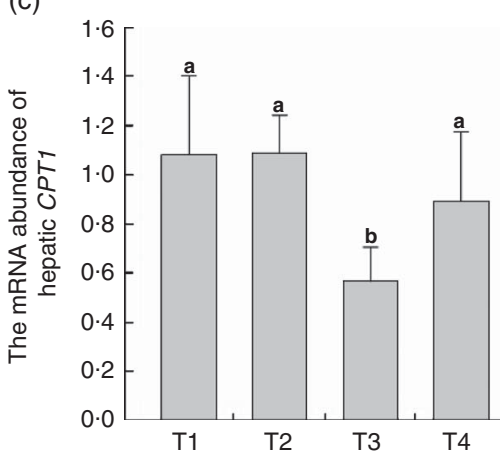

Fig. 4. (a) Effect of betaine on the concentration of carnitine palmitoyltransferase 1 (CPT1) in the liver. (b) Effect of betaine on the gene expression of PPARa in the liver. (c) Effect of betaine on the gene expression of CPT1 in the liver. Values are means $(n 7)$, with standard deviations represented by vertical bars. ${ }^{\text {a,b,c,d }}$ Mean values with unlike letters were significantly different $(P<0.05)$. Groups: T1, basal diet; T2, basal diet with betaine administration; T3, high-fat diet; T4, high-fat diet with betaine administration.

administration had no apparent effect on the concentration of CPT1 in rats fed with basal diet.'

The titles of the $y$ axes in the Fig. 3(a) and Fig. 4(a) were incorrect. They should read: The concentration of hepatic BHMT (ng/g); The concentration of hepatic CPT1 (ng/g)
The authors sincerely apologise for this errors.

\section{Reference}

Xu L, Huang D, Hu Q, Wu J, Wang Y \& Feng J (2015) Betaine alleviates hepatic lipid accumulation via enhancing hepatic lipid export and fatty acid oxidation in rats fed with a high-fat diet. British Journal of Nutrition, 1-9. 